\title{
Surface plasmon resonance in chalcogenide glass-based optical system
}

J. Le Person ${ }^{a}$, F. Colas $^{a}{ }^{*}{ }^{*}$, C. Compère ${ }^{a}$, M. Lehaitre ${ }^{a}$, M.-L. Anne ${ }^{b}$, C. Boussard-Plédel ${ }^{b}$, B. Bureau $^{b}$, J.-L. Adam ${ }^{b}$, S. Deputier ${ }^{c}$ and M. Guilloux-Viry ${ }^{c}$

a IFREMER, Service Interfaces et Capteurs, BP 7029280 Plouzané, France

${ }^{\mathrm{b}}$ Equipe Verres et Céramiques UMR CNRS 6226 Sciences chimiques de Rennes, Université de Rennes 1 , Campus de Beaulieu, 35042 Rennes cedex, France

${ }^{c}$ Equipe Chimie du Solide et Matériaux UMR CNRS 6226 Sciences chimiques de Rennes, Université de Rennes

1, Campus de Beaulieu, 35042 Rennes cedex, France

*: Corresponding author : F. Colas, email address : Florent.Colas@ifremer.fr

\begin{abstract}
:
The surface plasmon resonance phenomenon has been studied in a chalcogenide glass-based optical system. IR transmission properties of these materials combined to their high refractive indices lead to advantageous properties for sensing. In this study, numerical simulations have been carried out to investigate the potentialities of sulfide glass from the GeGaSbS system as a coupling prism material. Then, an angular modulation SPR biosensor has been set up in the Kretschmann-Raether arrangement. Experimental data are consistent with numerical calculation and the detection limit of the sensor is $3 \times 10-5 \mathrm{RIU}$. These preliminary results are promising. Further investigations have to be carried out to confirm the great potentialities of those materials for SPR-based biosensor.
\end{abstract}

Keywords: Chalcogenide glass; Surface plasmon resonance; Biosensor; Infrared 


\section{Introduction}

The surface plasmon resonance (SPR) phenomenon is widely used in the field of optical sensors to probe refractive index variations of chemical or biological samples [1-2]. It arises from the interaction of the incident light with plasmonic waves propagating at a metal/dielectric interface. Usually, the light coupling is carried out through a high-refractive-index substrate in the Kretschmann-Raether arrangement [3].

Up to now, most of SPR sensors are made from silica-based glass, which restricts SPR sensors to operate in the visible light range. Plasmon parameters of excitation in the IR range are very different and lead to advantageous properties for sensing [4,5]. The sensitivity of the SPR-based sensors for IR wavelengths is greatly improved: the probe depth of the plasmons is increased to more than the micron [6] and reflectivity curves are narrower, leading to a more precise SPR-dip position determination [7].

SPR excitation with IR light involves the use of advanced materials transmitting in the IR range. Silicon prism based systems have demonstrated interesting capabilities [4,5]. For the first time, we report here the development of a SPR sensor taking advantages of chalcogenide glass [8] as coupling material.

The composition selected for this study was $\mathrm{Ge}_{20} \mathrm{Ga}_{5} \mathrm{Sb}_{10} \mathrm{~S}_{65}$, called 2S2G $[9,10]$. It presents a large transparency region from $600 \mathrm{~nm}$ in the visible to $11 \mu \mathrm{m}$ in the mid-IR. It exhibits high refractive index and a good fiber drawing capability thanks to its stability against crystallisation [9, 10].

Potentialities of 2S2G for SPR-sensing have been investigated by both numerical calculation using Fresnel coefficients and experimental studies.

\section{Materials and methods}

\section{Simulations:}

Prior to any experiment, computation studies were carried out to establish the conditions of plasmon excitation in the IR range with a 2S2G prism and to optimize the detection limit of an angular modulation SPR biosensor in the Kretschmann-Raether configuration.

The Rouard method [11] was used to figure out the reflectivity of a stack made of 2S2G glass/gold/ biomolecules/ water. For these simulations, the biomolecular layer was assumed to be homogeneous and was considered as a dielectric thin film of refractive index $n=1.44$ and whose thickness increased as biomolecules got fixed to the probes.

Optical properties of gold were taken from data of Ordal et al [12] and those of water from Hale and Query [13]. The dispersion relation of 2S2G glass was figured out from the following relation (2) [14]: $\mathrm{n}_{2 \mathrm{~S} 2 \mathrm{G}}(\lambda)=2.24047+2.693 .10^{-2} \lambda^{-2}+8.08 .10^{-3} \lambda^{-4}(1)$

with $\mathrm{n}$ the $2 \mathrm{~S} 2 \mathrm{G}$ refractive index and $\lambda$ the wavelength.

The optimization of the working wavelength and the gold thickness required the definition of a merit function, called intrinsic sensitivity $\left(\mathrm{S}_{\mathrm{i}}\right)$, similarly to [6]. It was defined by the change of the dip angular position $\left(\theta_{\mathrm{SPR}}\right)$ with regard to the change of the biomolecule layer thickness (e), divided by the dip "full width at half minimum" $\left(\delta \theta_{\mathrm{SPR}}\right)$ :

$\mathrm{S}_{\mathrm{i}}=\left.\frac{1}{\delta \theta_{\mathrm{SPR}}} \frac{\partial \theta}{\partial{ }^{2}}\right|_{\theta=\theta_{\min }}$

Let $\delta \theta_{\min }$ be the smallest measurable dip angular shift and $\delta e_{\min }$ the smallest detectable thickness change. $\delta \theta_{\min }$ can be approximated by:

$$
\left.\delta \theta_{\min } \mathrm{i} \ddot{\Theta^{\partial \theta}}\right|_{\theta=\theta_{\text {min }}} \delta \mathrm{e}_{\text {min }}
$$

The smallest dip shift that can be measured is of the order of magnitude $\delta \theta_{\text {SPR }}$. Assimilating $\delta \theta_{\min }$ to $\delta \theta_{\text {SPR }}$ leads to:

$$
\mathrm{S}_{\mathrm{i}}=\frac{1}{\delta \mathrm{e}_{\text {min }}}(4)
$$


Maximising $S_{i}$ then yielded to optimizing the detection limit.

\section{Sulphide-based glass prism}

The glass was prepared from elemental high-purity starting products: gallium and antimony, germanium and sulfur are $5 \mathrm{~N}$ products from Cerac, Umicore and Strem, respectively. The elements were weighed in a dry glove box, batched into a silica ampoule and pumped under vacuum to $10^{-4}$ Torr for few hours. After sealing, the ampoule was introduced in a rocking furnace and heated to 900 ${ }^{\circ} \mathrm{C}$ at $1^{\circ} \mathrm{C} \mathrm{min}^{-1}$ heating rate. The ampoule was maintained at that temperature for 12 hours. Then, the melt was quenched to room temperature by immersing the ampoule in water and the glass was annealed at the glass transition temperature $T_{g}$.

The 2S2G glass rod of $16-\mathrm{mm}$ diameter was cut and polished to the diameter in order to obtain a hemi-cylindrical prism of $10-\mathrm{mm}$ long. Such a shape was chosen to minimise the aberrations of a beam converging onto hemi-cylinder axis.

Gold thin film deposition

The sensing surface is a critical zone for the sensing system. The metal must have conduction band electrons able to be excited by the incoming light. Among metal candidates, gold is one of the more convenient because of its small dielectric constant, which results in a sharp and intense SPR signal. Chemical durability and resistance to oxidation are well-known qualities of this noble metal, sufficiently reactive to allow biological or chemical binding.

A $50 \mathrm{~nm}$-gold layer supporting the SPR effect was deposited on the sulphide glass shaped in hemicylindrical prism by d.c. sputtering from a metallic gold target. This deposition method has been chosen because it is well adapted to metal deposition. Moreover this method is expected to favour deposit adhesion thanks to the kinetic energy of the sputtered species that is higher than the one of species emitted in standard evaporation, which is also routinely used for metallic deposition.

Nevertheless, a preliminary study was required in order to determine the influence of deposition parameters, in particular applied voltage, argon pressure and target-substrate distance in order to precisely control the deposition rate. Indeed, optical properties of the sensor system needed to deposit a very thin metal layer. The optimization of deposition conditions was performed on standard oxide glass plates, before to deposit on 2S2G planar substrates and finally on 2S2G prisms. Then gold thin films were deposited at room temperature, under an argon pressure of $7.10^{-2} \mathrm{mbar}$, with an applied voltage of $-1.2 \mathrm{kV}$ keeping the target-substrate distance at $25 \mathrm{~mm}$. In these conditions, deposition rate was $4 \mathrm{~nm} / \mathrm{min}$. The optimized conditions of deposition have finally been successfully adapted to the hemi cylindrical prism, using a mask, in order to deposit the gold layer on the planar face of the prism only. In contrast with silica-based glasses, such as BK7 or SF11, no adhesion sub-layer, like chromium layer for instance, was required on 2S2G substrates. The better adherence of gold on 2S2G is likely due to the creation of Au-S bonds between sulphur atoms of the 2 S2G sulphide glass and gold. The microstructure and thickness of the gold layer were checked by scanning electron microscopy.

\section{Experimental set-up}

A SPR system has been set up in the Kretschmann-Raether arrangement (Fig. 1 and 2). The configuration is based on angular interrogation by a convergent beam. The gold-coated 2S2G prism is attached to a PEEK flow cell and placed on a rotary stage (Newport M-RS65) to adjust the angular prism position. The wavelength of the incident light is selected by passing an incoherent white light tungsten-halogen (Lot Oriel LSB 117) through a 1-meter-focal-length-monochromator (Jobin Yvon, HR1000). Then the monochromatic light is collimated and passed through a NIR polariser (Thorlabs) to select linear p-polarisation. A long-pass filter (Melles Griot) is used to avoid order superposition. The reflected light (from $750 \mathrm{~nm}$ to $2.2 \mu \mathrm{m}$ ) is visualised on a NIR vidicon camera (Hamamatsu C2741) connected to a 12-bit frame grabber (Imasys, PCvision+). A sharp decrease or dip of intensity on the reflected light beam can be observed for specific incident angles, highlighting the SPR phenomenon (Fig. 3).

The reflectivity is deduced by averaging the image rows. The correspondence between the pixel number and the angular position was figured out by calibrating the rotary stage with the dip shift on the camera. 


\title{
3. Results and discussion
}

\author{
Simulations:
}

Typical calculated resonance curves in a three-layer system (2S2G prism/ gold/ water) for two different wavelengths are shown on figure 3. The dip full width at half-minimum (fwhm) is $1^{\circ}$ at $0.7 \mu \mathrm{m}$ while at $1.5 \mu \mathrm{m}$ the fwhm is $0.074^{\circ}$. Increasing wavelengths leads to narrower reflectivity curves.

As shown in figure 3 , the resonance phenomenon is achieved at different angles for different wavelengths. Calculated values of angular dip position $\theta_{\mathrm{SPR}}$ for air, water and ethanol media depend on the wavelength. They respectively vary from $26.4^{\circ}$ to $26.5^{\circ}, 36.5^{\circ}$ to $37.4^{\circ}$ and $37.4^{\circ}$ to $38.5^{\circ}$ with a stabilisation of these values for increasing wavelengths.

The angular dip position $\theta_{\mathrm{SPR}}$ is also quite different according to the refractive index of the prism materials, $n_{p}$. Figure 4 presents calculated angular reflectivity curves at $0.9 \mu \mathrm{m}$ in a three-layer system (prism/gold/ water) for two different prism materials, 2S2G ( $\mathrm{n}_{2 \mathrm{~S} 2 \mathrm{G}}=2.286$ at $\left.\lambda=0.9 \mu \mathrm{m}\right)$ and $\mathrm{BK} 7$ $\left(n_{\mathrm{BK} 7}=1.509\right.$ at $\left.\lambda=0.9 \mu \mathrm{m}\right)$ glasses. $\theta_{\mathrm{SPR}}$ is $36.7^{\circ}$ for $2 \mathrm{~S} 2 \mathrm{G}$ glass while $\theta_{\mathrm{SPR}}$ is $64.9^{\circ}$ for $\mathrm{BK} 7$ at $0.9 \mu \mathrm{m}$ in pure water, traducing a decrease of $\theta_{\text {SPR }}$ with an increase of $n_{p}$. High-refractive index materials lead to low-incidence angles, which reduce the image aberration and hence increase spatial resolution [6]. Moreover, the large refractive index of the 2S2G glass enhances the narrowness of the reflectivity curve. The width of the resonance curve (fwhm) is $0.95^{\circ}$ for the BK7 glass while the reflectivity curve for the $2 \mathrm{~S} 2 \mathrm{G}$ glass is sharper with a fwhm of $0.32^{\circ}$.

Figure 5 shows wavelength dependence of the calculated intrinsic sensibility with a gold thin film thickness of $45 \mathrm{~nm}, 50 \mathrm{~nm}$ and $55 \mathrm{~nm}$. First, it should be noticed that the intrinsic sensitivity increases with the gold thickness. However, the dip contrast then decreases. $50 \mathrm{~nm}$ seems to be a good compromise. Figure 5 also shows that the system exhibits a maximum intrinsic sensitivity in the NIR. In fact, the longer the wavelength, the less mobile but the sharper the dip is. Taking into account the strong absorption of water between $2 \mu \mathrm{m}$ and $3 \mu \mathrm{m}$, the optimum working wavelength is about $1.5 \mu \mathrm{m}$.

Experimental:

Figure 6 presents experimental reflectivity graphs in the 2S2G prism/ gold/ ethanol configuration for different wavelengths. For increasing wavelengths, a shift of the angular position is observed, about $0.2^{\circ}$ for a wavelength shift of $580 \mathrm{~nm}$. This observation was predicted by numerical simulations and is close to the theoretical value $\left(0.15^{\circ}\right)$. At $1.2 \mu \mathrm{m}$, the fwhm is $0.32^{\circ}$ and decreases to $0.19^{\circ}$ at $1.78 \mu \mathrm{m}$. Calculated fwhm values are smaller, for instance $0.12^{\circ}$ at $1.2 \mu \mathrm{m}$. However, the decrease of the fwhm is consistent with numerical simulations and with data obtained by Patskovsky et al in silicon and in BK7 [5]. It reveals a higher field enhancement close to the surface for higher wavelengths [4] and a larger probe depth, which can be used for remote sensing [5].

The SPR phenomenon is achieved experimentally at different angles of incidence for air, water and ethanol, respectively $22.8^{\circ}, 34.4^{\circ}$ and $35.4^{\circ}$ at $680 \mathrm{~nm}$. These results are slightly smaller than those obtained by numerical simulations. Theoretical SPR angles of $26.5^{\circ}, 37.7^{\circ}$ and $38.9^{\circ}$ at $680 \mathrm{~nm}$ for air, water and ethanol respectively are obtained. The graph on figure 7 represents theoretical and experimental reflectivity with regard to the incident angle for $\lambda=680 \mathrm{~nm}$ and water as sensing medium. The discrepancy between these two sets of data can be explained by real non-ideal conditions such as gold surface roughness and nonuniformity or image aberration.

Aqueous solutions of ethanol have been used to quantify the sensor resolution. From reflectivity curves, angular dip positions have been measured for various ethanol dilutions in distilled water, from $0 \%$ to $96 \%$ ethanol. The graph on figure 8 shows the dip shift with regard to the concentration of ethanol. For concentration less than about $40 \%$, it linearly increases. Then it reaches a maximum and finally slightly decreases for high concentrations. That behaviour is consistent with the change of refractive index of ethanol solution with the concentration $[15,16]$.

During the experiment, ethanol was detected in pure water at a concentration as low as $0.05 \%$. At $\lambda=589 \mathrm{~nm}$, the refractive index difference between pure water and a $5 \%$ ethanol solution is about $3 \times 10^{-3}$ RIU [16]. Sarov et al [17] demonstrated that the dispersion of ethanol and pure water are similar in the near infrared range. Then, the detection limit of our system can be estimated to $3 \times 10^{-5} \mathrm{RIU}$, which is comparable to those obtained with other systems [18, 19].

The detection limit of the sensor could be improved by the use of a more powerful source. In fact, as it can be noticed on figure 2, the further the camera from the lens, the larger but the dimmer the image 
is. A more powerful source would enable us to work with a larger and more contrasted image. It would then be possible to detect smaller dip shift.

\section{Conclusion}

A new SPR sensor has been developed using a chalcogenide glass as a coupling prism material in the Kretschmann-Raether arrangement. It has been demonstrated that IR transmission properties of chalcogenide glass combined to their high refractive indices led to narrower reflectivity curves and a more precise SPR-dip position determination. Experimental data are consistent with theoretical calculations and a sensor detection limit of $3 \times 10^{-5} \mathrm{RIU}$ was demonstrated. Chalcogenide glasses could then be great candidates for the development of SPR sensor in infrared light thanks to their optical properties and to their large type of coupling configuration possibilities, like prism, fibre and planar waveguide coupling, allowing the development of miniaturised SPR-sensors.

\section{Acknowledgments}

This work was funded by the Brittany region (PRIR BIRMA). 


\section{References}

[1] J. Homola, S.S. Yee, D. Myszka, Surface Plasmon Resonance Biosensors, in: F.S. Ligler and C.A.R. Taitt (Ed.s), Elsevier, The Netherlands, 2002, pp. 207-251.

[2] D.R. Shankaran, K.V. Gobi, N. Miura, Recent advancements in surface plasmon resonance immunosensors for detection of small molecules of biomedical, food and environmental interest, Sens. Actuators B 121 (2007) 158-177.

[3] E. Kretschmann, H. Raether, Radiative decay of non radiative surface plasmon excited by light, Z. Naturforsch. A 23 (1968) 2135-2136.

[4] G. Brink, H. Sigl, E. Sackmann, Near-infrared surface plasmon resonance in silicon based sensor: new opportunities in sensitive detection of biomolecules from aqueous solution by applying microstep for discriminating specific and non specific binding, Sens. Actuators B 24-25 (1995) 756-761.

[5] S. Patskovsky, A. V. Kabashin and M. Meunier, Properties and sensing characteristics of surfaceplasmon resonance in infrared light, J. Opt. Soc. Am., 20, 8 (2003) 1644-1650.

[6] K. Johansen, H.Arwin, I. Lundström and B. Liedberg, Imaging surface plasmon resonance sensor based on multiple wavelengths: sensitivity considerations, Rev. Sci. Instrum., 71 (2000) 3530-3538.

[7] B. P. Nelson, A.G. Frutos, J.M. Brockman and R.M. Corn, Near-infrared surface plasmon resonance measurements of ultrathin films, Anal. Chem. 71 (1999) 3928-3934.

[8] B. Bureau, X.H. Zhang, F. Smektala, J-L Adam, J. Troles, H-L. Ma, C. Boussard-Plédel, J. Lucas, P. Lucas, D. Le Coq, M.R. Riley, J.H. Simmons, Recent advances in chalcogenide glasses, J. NonCryst. Solids $345 \& 346$ (2004) 276-283.

[9] Y. Guimond, J-L. Adam, A.M. Jurdyc, J. Mugnier, B. Jacquier, X.H. Zhang, Dy ${ }^{3+}$-doped stabilized GeGaS glasses for $1.3 \mu \mathrm{m}$ optical fiber amplifiers, Opt. Mater. 12 (1999) 467.

[10] J. Le Person, F. Smektala, T. Chartier, L. Brilland, T. Jouan, J. Troles, and D. Bosc, Light guidance in new chalcogenide holey fibres from GeGaSbS glass, Mater. Res. Bull. 41 (2006) 13031309.

[11] P. Rouard, Annales de Physique, 7 (1937) 291.

[12] M. A. Ordal, L. L. Long, R. J. Bell, S. E. Bell, R.W. Alexander Jr., C.A. Ward, Optical properties of the metals $\mathrm{Al}, \mathrm{Co}, \mathrm{Cu}, \mathrm{Al}, \mathrm{Fe}, \mathrm{Pb}, \mathrm{Ni}, \mathrm{Pd}, \mathrm{Pt}, \mathrm{Ag}, \mathrm{Ti}$ and $\mathrm{W}$ in the infrared and far infrared, Appl. Opt. 22, 7 (1983) 1099-1118.

[13] G.M. Hale and M.R. Querry, Optical constants of water in the $200 \mathrm{~nm}$ to $200 \mu \mathrm{m}$ wavelength region, Appl. Opt. 12, 3 (1973) 555-563.

[14] J. Le Person, Thèse de l'Université de Rennes 1, 10 septembre 2004, Verres de sulfure: Spectroscopie des ions de terres-rares, fibres microstructurées et nouvelles compositions.

[15] T. A. Scott, Refractive index of ethanol-water mixtures and density and refractive index of ethanolwater-ethyl ether mixtures, Jr. J. Phys. Chem. 50 (1946) 406-412.

[16] D. R. Lide (Ed.) 2001-2002 Handbook of chemistry and physics $82^{\text {nd }}$ edition, 8-62.

[17] Y. Sarov, S. Sainov, I. Kostic, V. Sarova, S. Mitkov, Automatic VIS - near IR laser refractometer, Rev. Sci. Instrum., 25, 10 (2004) 3342-3344

[18] J. Homola, S. S. Yee, G. Gauglitz, Surface plasmon resonance sensors: review, Sens. Actuators B54 (1999) 3-15.

[19] J. Meléndez, R. C. Carr, D. Bartholomew, H. Taneja, S. Yee, C. Jung, C. Furlong, Developpment of a surface plasmon resonance sensor for commercial applications, Sens. Actuators B 38-39 (1997) 375-379. 


\section{Figures}

p-polarised incoming light Reflected light

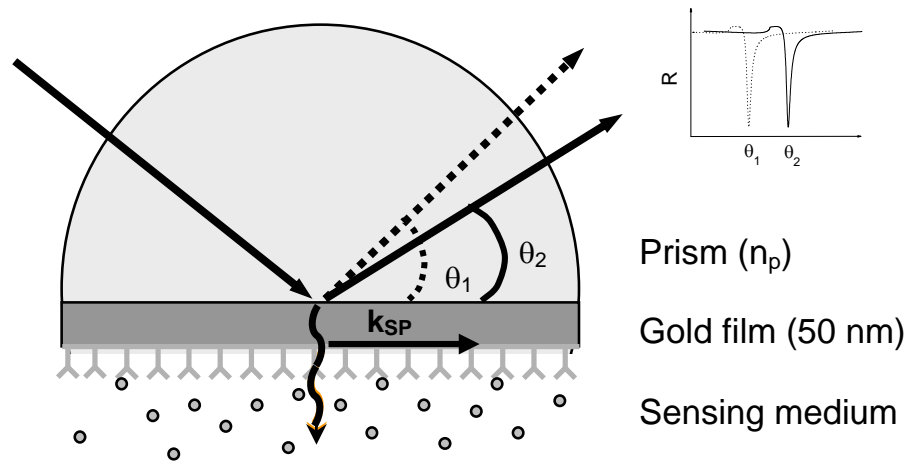

Evanescent wave

Figure 1:

Schematic illustration of surface plasmon resonance in Kreschtmann-Raether arrangement (prism/ gold/ biomolecular layer/ sensing medium).
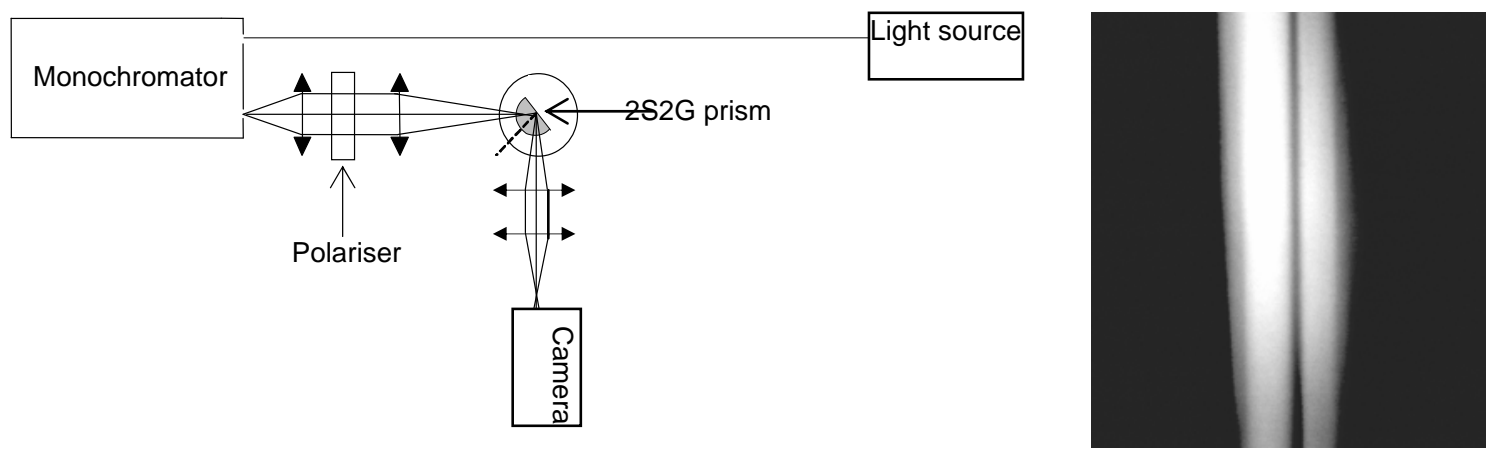

Figure 2:

(a) Experimental set-up, on the left

(b) Dip of intensity on the reflected p-polarised light beam, imaged on the near-IR camera, on the right. 




Figure 3:

Calculated reflectivity curves for two wavelengths, $0.7 \mu \mathrm{m}$ and $1.5 \mu \mathrm{m}$, in the case of a three-layer system (2S2G prism/ gold/ water).

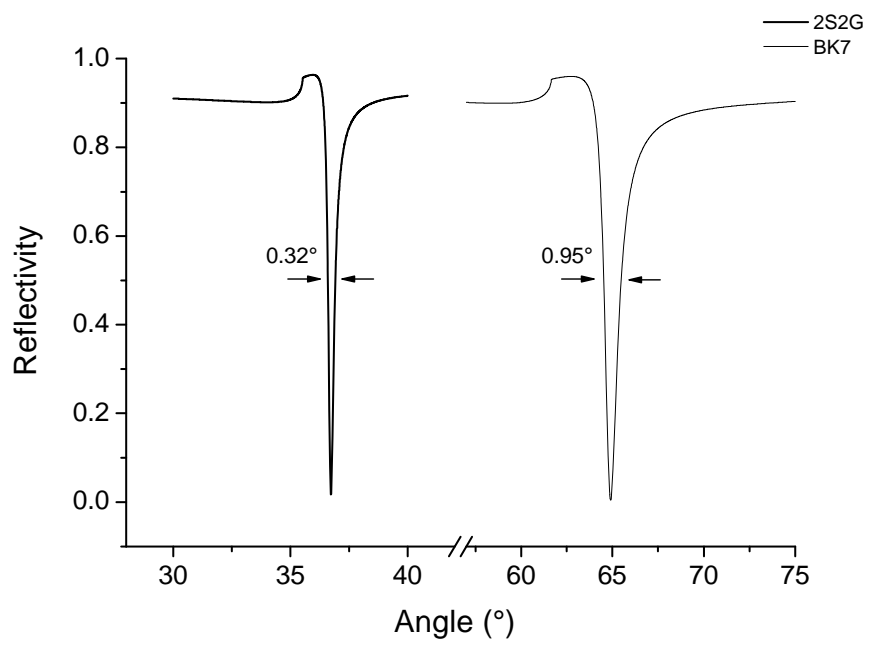

Figure 4:

Calculated reflectivity curves in a three-layer system (prism/gold/ water) for two different prism materials, $2 \mathrm{~S} 2 \mathrm{G}$ and BK7 glass, at $0.9 \mu \mathrm{m}$. 


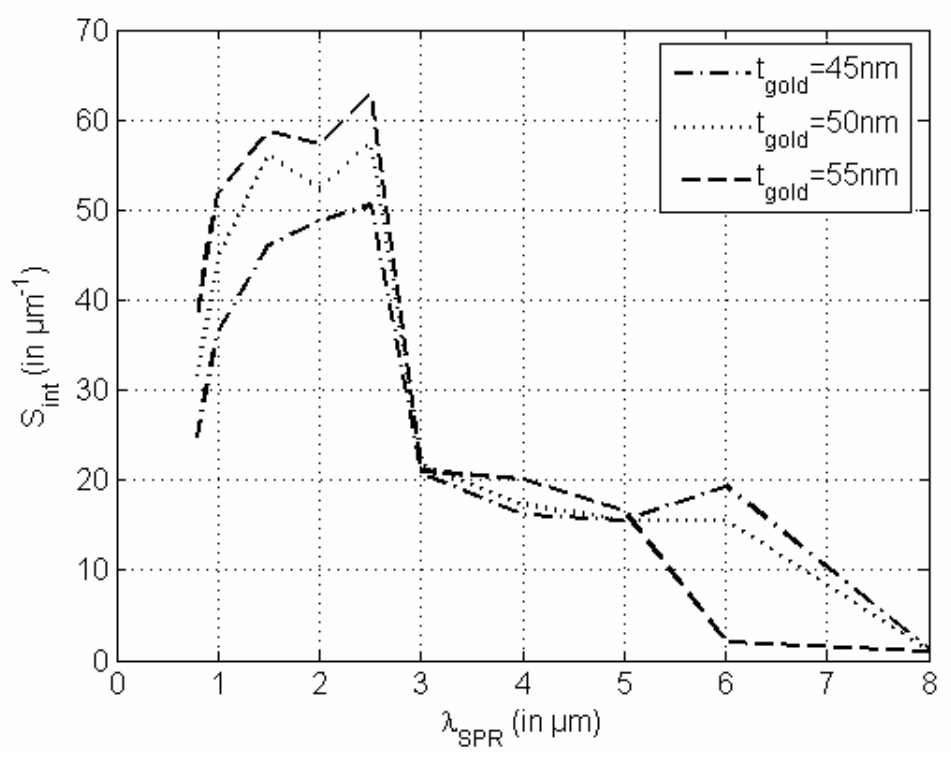

Figure 5:

Wavelength dependence of intrinsic sensibility in a four-layer system (2S2G glass/ gold/ protein layer/ water).

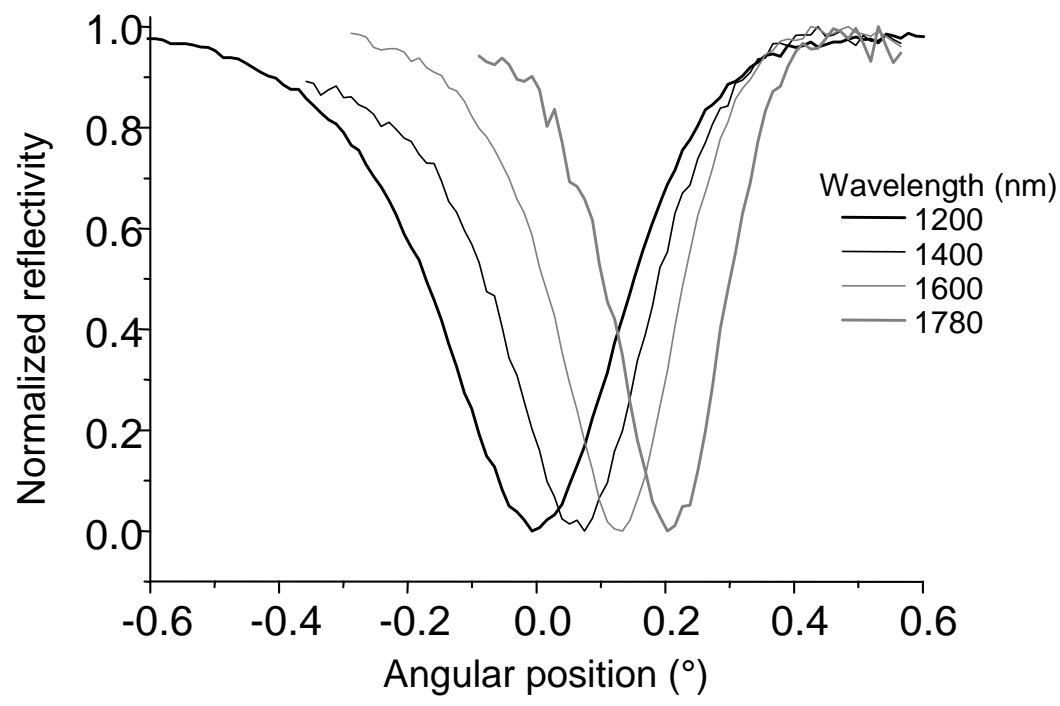

Figure 6:

Experimental reflectivity curves in the $2 \mathrm{~S} 2 \mathrm{G}$ prism/gold/ ethanol configuration for different wavelengths. 


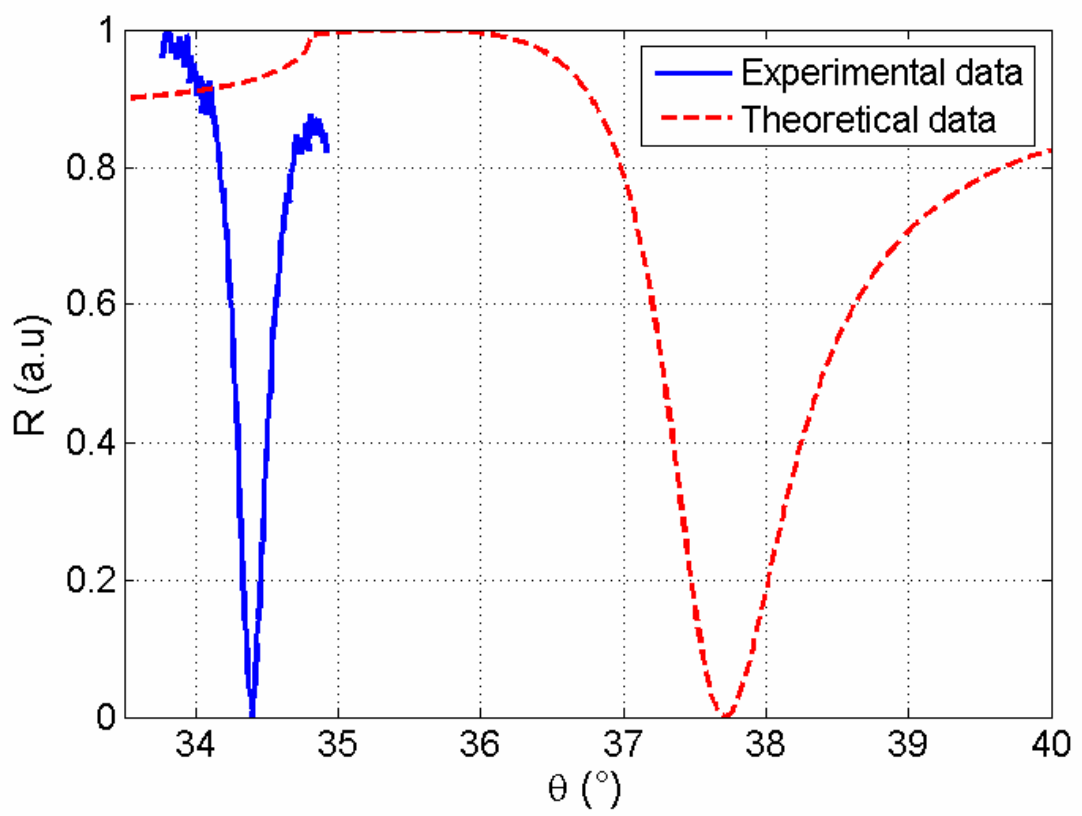

Figure 7:

Experimental and theoretical reflectivity curves in the $2 \mathrm{~S} 2 \mathrm{G}$ prism/ gold/ water configuration at $680 \mathrm{~nm}$.

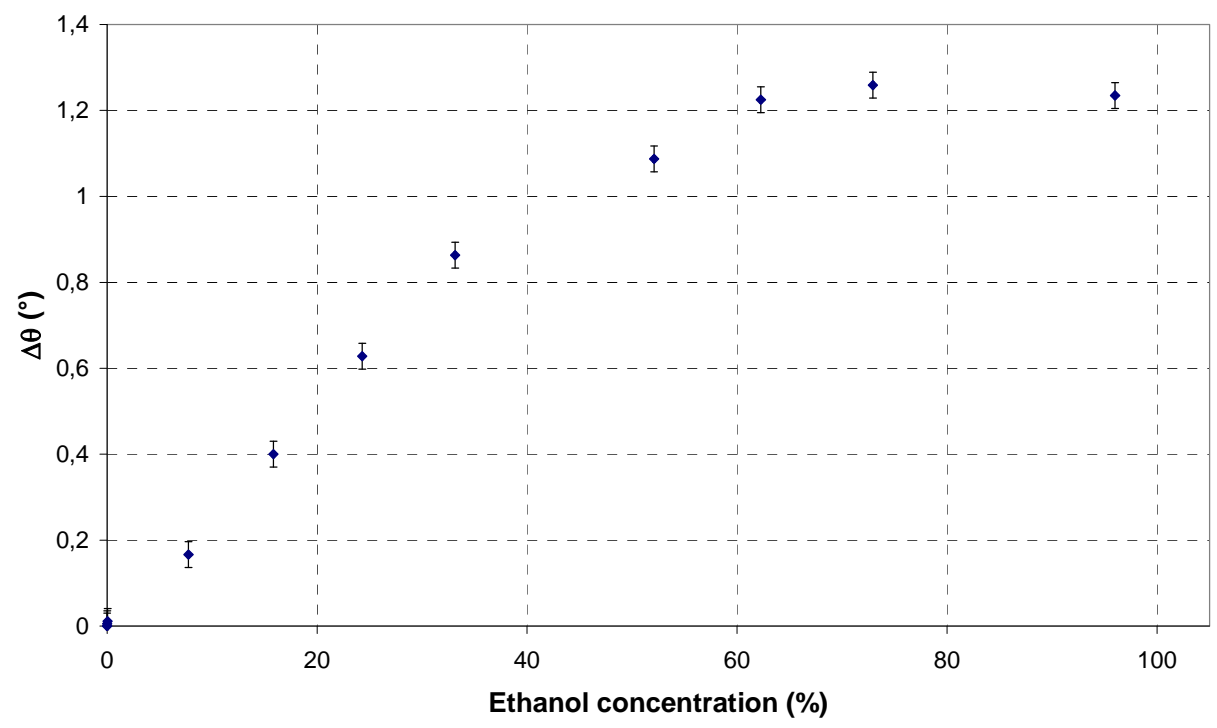

Figure 8:

Dip shift with regard to ethanol concentration, at $1.6 \mu \mathrm{m}$. 


\section{Biographies}

J. Le Person received the Ph. D. degree in materials chemistry from the University Rennes I (France) in 2004, for her work on infrared glasses.

Se then spent two years as post-doctoral scientist in Ifremer (France), working on SPR-based optical biosensor.

Currently, she is involved in high power fiber lasers in a new company, MANLIGHT, France.

F. Colas received an engineering degree in Optics at SupOptique (France) and an M. Sc. degree in Optics and Photonics at Imperial College (UK) in 2004. He holds a position as Engineer at Ifremer, where he works on optical sensor for the marine environment.

C. Compère received her Ph. D. in Chemistry from Louis Pasteur University (Strasbourg, France) in 1984. After 2 years as post-doctoral researcher for the National Sciences and Engineering Research Council of Canada at the National Research Council Canada (Bourcheville, Quebec), she accepted a position as a research scientist at the NRC. Her research has been primarily in the field of electrochemistry and corrosion and in 1990, she has continued on this field of study at the Laval's Department of Mining, Metallurgical and Material Engineering, Quebec, Canada. In 1992, she joined Ifremer and she is presently responsible for the Interfaces and Sensors laboratory at Ifremer, France. The laboratory conducts research on biosensors, optical sensors for in situ monitoring in natural seawater, surface modifications, characterization of biomolecules at interfaces and the understanding of biofilm and biofouling formation in order to develop new antifouling treatments.

\section{Michel Lehaitre:}

Engineer in optoelectronic, he has been working in the field of marine instrumentation, and more specifically in the development of underwater sytems for more than 30 years. He has been participating in lot of oceanographic cruises in open sea for deep hydrothermal studies and for coastal water monitoring as well. He has got lot of experience in underwater optics particularly for suspended matter analysis through scattering and spectal absorption process. He has been in charge of the development of an in situ particle size analyser and more recently for deep ocean Raman SERS applications.

M.-L. Anne, received a Ph. D. in Chemistry in September 2007 from the University of Rennes, France. Her thesis is entitled "Waveguides made of chalcogenide glasses for infrared detection of biomolecules". Her main research activities deal with the synthesis and characterization of chalcogenide glasses and their elaboration as sensors (fibers and thin films) for biomedical and environmental applications.

Since October, she is in a post-doc position in the "Glass and Ceramics Laboratory" in collaboration with the ESA to develop single mode fibers for spatial applications.

C. Boussard-Plédel, Ph. D. in Chemistry, with a thesis focused on glasses in the system B-O-F. Since 1999, she is engineer at the CNRS, in the Glasses and Ceramics group of the Chemical Sciences Center in Rennes, France.

Her research activities deals with the elaboration and characterization of infrared bulk glasses (chalcogenide, fluoride), the elaboration of infrared fibers (multimode, single mode) and the development of infrared sensors with applications in biomedical, environmental and spatial areas.

She is involved in several international research programs. She is author and co-author of about 40 publications and 1 patent.

B. Bureau, Ph. D, "Local order investigations in fluoride glasses by multinuclear solid state NMR", defended on 9th December 1998 at the University of Le Mans, France. From 1999 to 2006, he was Associated Professor in the "Glasses and Ceramics Laboratory » of the University of Rennes, France. He has an Accreditation to supervise research. Since 2006, he is Full Professor in charge of the passive optic group working on infrared optical fibers for sensors and responsible of the Master 1 in chemistry (4th degree) of the University of Rennes. He is also a solid state NMR expert. He is responsible of several international research programs. He is author and co-author of more than 60 publications and delivered 20 invited lectures. 
J.-L. Adam received a Ph. D. degree in chemistry from the University of Rennes (France) in 1983. After a one-year postdoctoral appointment at Oklahoma State University, Stillwater, he joined the Centre National de la Recherche Scientifique (CNRS) in France in 1985. Since then, his research has been devoted essentially to the chemistry of non-oxide glasses and to the spectroscopy of optically active ions for telecommunications and solid-state laser systems, including research on optical fibers and waveguides. In 1989, he was a research associate at IBM-Almaden Research Center (San Jose, USA), conducting research on glasses for optical memories. Presently, he is a research director at CNRS and Vice-Director of the Chemical Sciences Center in Rennes. He has authored or co-authored more than 160 papers and book chapters and delivered 40 invited lectures at international conferences.

S. Députier received the Ph. D. degree in chemistry from the University of Rennes 1, Rennes, France, in 1992. Since 1994, she has been an Assistant Professor at Laboratoire de Chimie du Solide et Inorganique Moléculaire, from University of Rennes 1, Rennes, France. Her research activities currently develop in solid state chemistry (especially phase diagrams study) and in thin films growth and structural characterization field.

M. Guilloux-Viry received the Ph. D. degree in physics from the University of Rennes 1 , Rennes, France, in 1991. She was researcher at Centre National de la Recherche Scientifique (CNRS) from 1991 to 2005 at Laboratoire de Chimie du Solide et Inorganique Moléculaire, from University of Rennes 1. She became Professor in 2005 at Unité Sciences Chimiques, UMR 6226 CNRS - University of Rennes 1 . She is now in charge of managing the group "Solid State Chemistry and Materials". Her main research field concerns synthesis and characterization of thin films, in particular epitaxial growth (by pulsed laser deposition and sputtering) and characterization of thin films of complex materials (such as perovskites-like oxides, presenting superconducting or ferroelectric behaviours, and Chevrelphase type sulfides). 